\title{
Article \\ Electromagnetic Form Factor of Doubly-Strange Hyperon
}

\author{
Xiongfei Wang $1,2, * \mathbb{1}$ and Guangshun Huang $3,4, *$ (i) \\ 1 School of Physical Science and Technology, Lanzhou University, Lanzhou 730000, China \\ 2 Lanzhou Center for Theoretical Physics, Frontiers Science Center for Rare Isotopes and Key Laboratory of \\ Theoretical Physics of Gansu Province, Lanzhou University, Lanzhou 730000, China \\ 3 Department of Modern Physics, University of Science and Technology of China, Hefei 230026, China \\ 4 State Key Laboratory of Particle Detection and Electronics, Hefei 230026, China \\ * Correspondence: wangxiongfei@lzu.edu.cn (X.W.); hgs@ustc.edu.cn (G.H.)
}

check for updates

Citation: Wang, X.; Huang, G. Electromagnetic Form Factor of Doubly-Strange Hyperon. Symmetry 2022, 14, 65. https://doi.org/ 10.3390/sym 14010065

Academic Editors: Monica Bertani, Simone Pacetti, Alessio Mangoni and Charalampos Moustakidis

Received: 28 November 2021 Accepted: 25 December 2021

Published: 3 January 2022

Publisher's Note: MDPI stays neutral with regard to jurisdictional claims in published maps and institutional affiliations.

Copyright: (C) 2022 by the authors. Licensee MDPI, Basel, Switzerland. This article is an open access article distributed under the terms and conditions of the Creative Commons Attribution (CC BY) license (https:// creativecommons.org/licenses/by/ $4.0 /)$.

\begin{abstract}
The standard model of particle physics is a well-tested theoretical framework, but there are still some issues that deserve experimental and theoretical investigation. The $\Xi$ resonances with strangeness $S=-2$, the so-called doubly-strange hyperon, can provide important information to further test the standard model by studying their electromagnetic form factors, such as probing the limitation of the quark models and spotting unrevealed aspects of the QCD description of the structure of hadron resonances. In this work, we review some recent studies of the electromagnetic form factors on doubly-strange hyperons in pair production from positron-electron annihilation experiment.
\end{abstract}

Keywords: electromagnetic form factor; Born cross section; hyperon pair production

\section{Introduction}

Electromagnetic form factors (EMFFs) parameterizing the inner structure and dynamics of hadrons are fundamental observables of nucleons and hyperons to understand the QCD effects in hadronic resonances [1-6]. In the 1960s, Cabibbo et al. [7] first proposed that time-like EMFFs can be studied on $e^{+} e^{-}$collider experiments by measuring the hadron pair production cross sections, such as the exclusive hyperon cross section. Figure 1 shows the lowest-order process for the hyperon-antihyperon $(H \bar{H})$ production in $e^{+} e^{-}$annihilation.

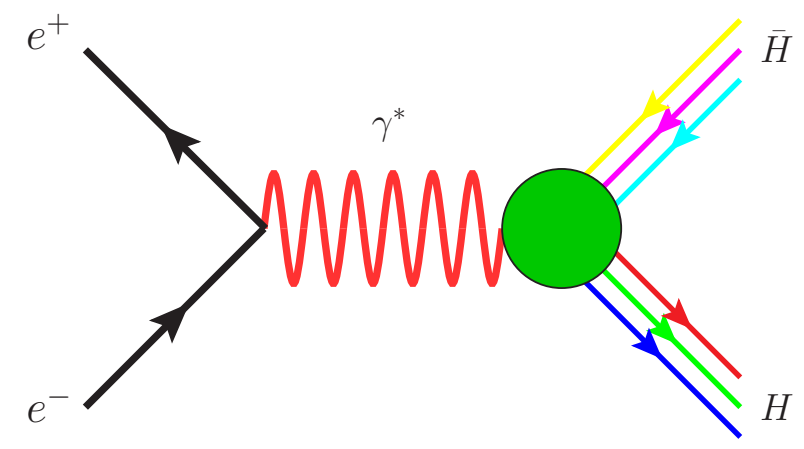

Figure 1. Lowest-order Feynman diagram for the $e^{+} e^{-} \rightarrow H \bar{H}$ process.

Assuming spin-1/2 hyperon pair production is dominated by one virtual photon exchange, the differential cross section for the $e^{+} e^{-} \rightarrow H \bar{H}$ process in the $e^{+} e^{-}$center-ofmass (c.m.) frame can be expressed in terms of the EMFFs [7] $G_{E}(s)$ and $G_{M}(s)$ as:

$$
\begin{gathered}
\frac{d \sigma^{B}(s)}{d \Omega}=\frac{\alpha^{2} \beta C}{4 s}\left[\left|G_{M}(s)\right|^{2}\left(1+\cos ^{2} \theta\right)+\frac{1}{\tau}\left|G_{E}(s)\right|^{2} \sin ^{2} \theta\right], \\
G_{E}(s)=F_{1}+\tau F_{2}
\end{gathered}
$$




$$
G_{M}(s)=F_{1}+F_{2},
$$

where $F_{1}$ and $F_{2}$ are the Dirac's and Pauli's form factors [8], respectively, $\alpha$ is the finestructure constant, $s$ is the square of the c.m. energy, $\beta=\sqrt{1-4 m_{H}^{2} c^{4} / s}$ is the velocity of the outgoing hyperon, and $m_{H}$ is the mass of the hyperon, $\tau=\frac{s}{4 m_{H}^{2} c^{4}}$. The Coulomb factor $\mathcal{C}$ parameterizes the electromagnetic interaction of point-like fermions in the final state [9-11], and is unity for neutral hyperon, while for a charged one, $\mathcal{C}=y /\left(1-e^{-y}\right)$ with $y=\pi \alpha \sqrt{1-\beta^{2}} / \beta$ [12-14]. In ref. [15], $\mathcal{C}$ is parameterized as a product of an enhancement factor $\mathcal{E}$ and a resummation factor $\mathcal{R}$, i.e., the so-called Sommerfeld-Schwinger-Sakharov rescattering formula: $\mathcal{C}=\mathcal{E} \times \mathcal{R}$ with $\mathcal{E}=\pi \alpha / \beta$. In the limit of $\beta \rightarrow 0$, and the factor $\beta$ due to the limitation of the phase space is canceled and results in a nonzero cross section at the production threshold. By integrating over a full solid angle for Equation (1), we can obtain the Born cross section as:

$$
\sigma^{B}(s)=\frac{4 \pi \alpha^{2} \beta \mathcal{C}}{3 s}\left[\left|G_{M}(s)\right|^{2}+\frac{1}{2 \tau}\left|G_{E}(s)\right|^{2}\right],
$$

and then, we can define the effective form factor $(E F F) G_{\text {eff }}(s)[28]$ by:

$$
\left|G_{\text {eff }}(s)\right|=\sqrt{\frac{2 \tau\left|G_{M}(s)\right|^{2}+\left|G_{E}(s)\right|^{2}}{2 \tau+1}},
$$

where the EMFFs $G_{E}(s)$ and $G_{M}(s)$ can be expressed by:

$$
\begin{gathered}
\left|G_{M}(s)\right|^{2}=\frac{2 \tau+1}{2 \tau+R^{2}}\left|G_{e f f}(s)\right|^{2}, \\
\left|G_{E}(s)\right|^{2}=\frac{R^{2}(2 \tau+1)}{2 \tau+R^{2}}\left|G_{e f f}(s)\right|^{2},
\end{gathered}
$$

with a definition of the ratio between $G_{M}(s)$ and $G_{E}(s)$ as:

$$
R=\left|\frac{G_{E}(s)}{G_{M}(s)}\right| .
$$

According to Equations (4) and (5), the EFF can be further written as:

$$
\begin{aligned}
\left|G_{e f f}(s)\right| & =\sqrt{\frac{3 s \sigma^{B}}{4 \pi \alpha^{2} \beta \mathcal{C}\left(1+\frac{1}{2 \tau}\right)}} \\
& =\sqrt{\frac{3 s \sigma^{B}}{4 \pi \alpha^{2} \beta \mathcal{C}\left(1+2 m_{H}^{2} c^{4} / s\right)}} .
\end{aligned}
$$

Additionally, Equation (1) can be written as:

$$
\frac{d \sigma^{B}(s)}{d \cos \theta} \propto\left[\left(1+\cos ^{2} \theta\right)+\frac{R^{2}}{\tau} \sin ^{2} \theta\right] \propto\left[1+\frac{\tau-R^{2}}{\tau+R^{2}} \cos ^{2} \theta\right],
$$

and Equation (10) can be further expressed to be a simple form as:

$$
\frac{d \sigma^{B}(s)}{d \cos \theta} \propto 1+\eta \cos ^{2} \theta
$$

with

$$
\eta=\frac{\tau-R^{2}}{\tau+R^{2}}
$$

where the ratio $\mathrm{R}$ can be further expressed to be 


$$
R=\sqrt{\frac{\tau(1-\eta)}{1+\eta}},
$$

where $\eta$ is the angular distribution parameter and in principle it can be determined by studying angular distribution.

For time-like regions, the EMFFs can be complex, i.e.,

$$
\begin{aligned}
G_{M}(s) & =\left|G_{M}(s)\right| e^{i \Phi_{M},} \\
G_{E}(s) & =\left|G_{E}(s)\right| e^{i \Phi_{E},}
\end{aligned}
$$

from which the relative phase can be defined: $\Delta \Phi=\Phi_{E}-\Phi_{M}$. Then, the electric form factor $G_{E}(s)$ can be expressed in terms of the magnetic form factor as:

$$
G_{E}(s)=R e^{i \Delta \Phi}\left|G_{M}(s)\right| .
$$

Here, $\Delta \Phi$ is the relative phase between $G_{E}(s)$ and $G_{M}(s)$, which corresponds to the hyperon polarization if a non-zero relative phase is observed. Thus, if we have enough statistics, the relative phase can be determined combined with the study of the hyperon polarization. Similarly, the EMFFs can be measured separately using the measured relative phase combined with Equation (5).

In the context of QCD and the quark-gluon structure of hadrons, it is particularly interesting to measure the EMFFs of nucleons and strange hyperons $(\Lambda, \Sigma, \Xi, \Omega)$, which is expected to reveal the aspects of the QCD description of the hyperon structure. In experiments, a large amount of research has been carried out regarding nucleon and strange hyperon EMFFs in the time-like $\left(q^{2}>0\right)$ momentum transfer regions [16]. Among them, the proton is a stable particle, and also can be available as a target to study its EMFFs by means of scattering experiments in the space-like $\left(q^{2}<0\right)$ momentum transfer. Hyperons, which are slightly different from protons, are difficult to study in the space-like region since they are unstable. In other words, hyperon targets are unfeasible and the quality of hyperon beams is in general not sufficient. Time-like EMFFs therefore offer the best opportunity to study hyperon structure. The $\Xi^{0}$ and $\Xi^{-}$hyperons are members of the SU(3) octet [17], similar to the nucleon, as shown in Figure 2. Understanding their structure is crucial to obtaining a complete picture of how quarks and gluons form hyperons.

In this article, we review the recent studies of EMFFs on doubly-strange hyperons using the pair production near threshold and above open charm threshold reported by the BESIII experiment $[18,19]$ and CLEO-c experiment $[20,21]$. These results provide useful and important information to understand the internal structure and production mechanism of doubly-strange hyperons, and even offer an extra dimension that probes the limitation of the quark models and spots unrevealed aspects of the QCD description of the structure of hadron resonances.

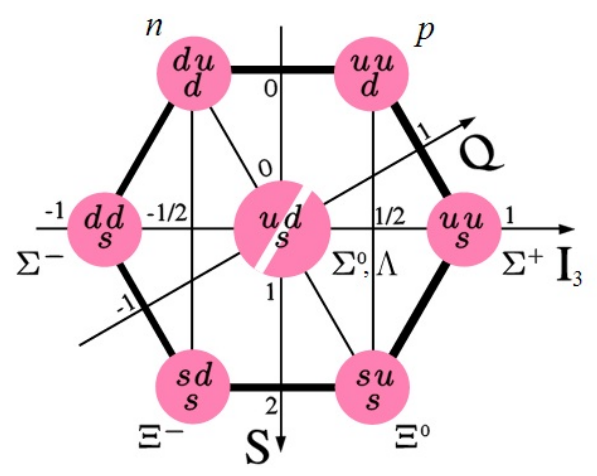

Figure 2. Baryon octet $[4,17]$. 


\section{The $\Xi$ EMFFs near Threshold}

In the past decades, a few experiments have observed non-vanishing cross sections or enhancements near threshold in the production cross section of nucleon pairs at $e^{+} e^{-}$ colliders, which have attracted much interest and driven many theoretical studies. In the context of QCD and even our understanding of the quark-gluon structure of hadrons, the study of EMFFs near the hyperon pair production threshold is particularly interesting for probing these anomalous phenomena [22-25]. Recently, the BESIII collaboration performed measurements of Born cross sections and EFFs for the processes $e^{+} e^{-} \rightarrow \Xi^{-} \bar{\Xi}^{+}$ and $\Xi^{0} \bar{\Xi}^{0}$ based on a single hyperon tag method [26-32] using about 360 to $500 \mathrm{pb}^{-1}$ data events collected at c.m. energies between 2.644 and $3.080 \mathrm{GeV}[33,34]$. Figure 3 shows the two-dimensional distribution of $M_{\pi \Lambda}$ versus $M_{\pi \Lambda}^{\text {recoil }}$ for the sum of all energy points, where anti-hyperon candidates are extracted from the mass recoiling against the reconstructed $\pi \Lambda$ system,

$$
M_{\pi \Lambda}^{\text {recoil }}=\sqrt{\left(\sqrt{s}-E_{\pi \Lambda}\right)^{2}-\left|\vec{p}_{\pi \Lambda}\right|^{2}}
$$

Here $E_{\pi \Lambda}$ and $\vec{p}_{\pi \Lambda}$ are the energy and momentum of the selected $\pi \Lambda$ candidate in the c.m. system. Clear signals for $\Xi^{-} \bar{\Xi}^{+}$and $\Xi^{0} \bar{\Xi}^{0}$ productions can be seen around the $\Xi$ nominal mass near threshold. Figure 4 shows the comparisons of the measured Born cross sections and EFFs between both modes from 2.6 to $3.1 \mathrm{GeV}$.
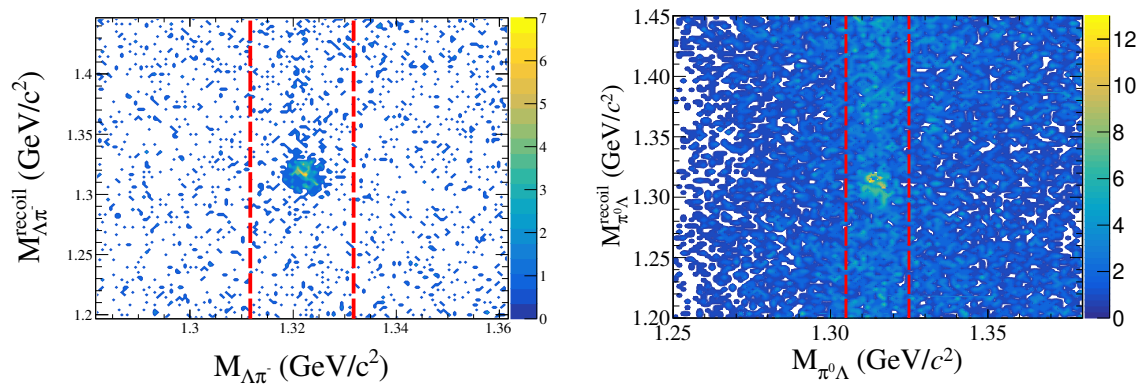

Figure 3. Distribution of $M_{\pi \Lambda}$ versus $M_{\pi \Lambda}^{\text {recoil }}$ for all energy points from data [33,34]. The dashed lines denote the $\Xi$ signal region.

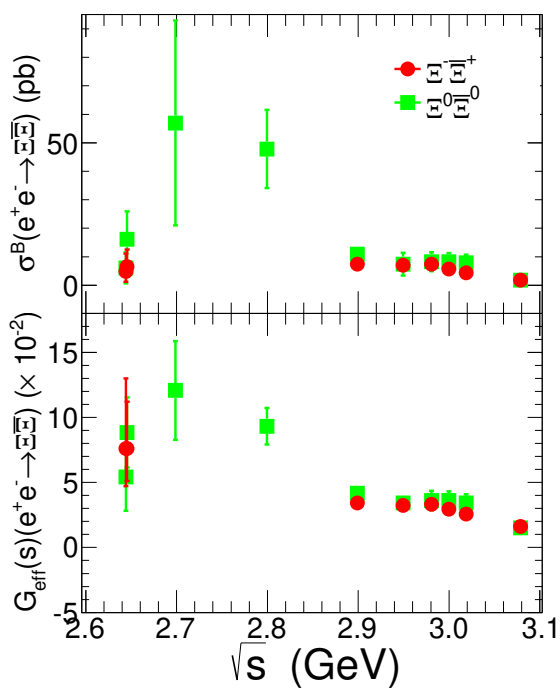

Figure 4. Comparisons of Born cross sections (top) and EFFs (bottom) between the $e^{+} e^{-} \rightarrow \Xi^{-} \bar{\Xi}^{+}$ mode and the $e^{+} e^{-} \rightarrow \Xi^{0} \bar{\Xi}^{0}$ mode from 2.6 to $3.1 \mathrm{GeV}$, where the uncertainties include both statistical and systematical contributions. 
To understand well the doubly-strange hyperon production near threshold, a pQCDdriven energy power function [35] is used to describe the measured Born cross section as:

$$
\sigma^{B}=\frac{c_{0} \cdot \beta}{\left(\sqrt{s}-c_{1}\right)^{10}}
$$

where $c_{0}$ is the normalization factor, and $c_{1}$ is the mean effect of possible intermediate states. This model has also been applied successfully for the studies of other hyperon pair productions in $e^{+} e^{-}$annihilation [24,36]. However, it is seen that the fit with the pQCD function only cannot describe the data points around $3.0 \mathrm{GeV}$ well. To validate and accommodate a possible structure in this region, the other fit by assuming a coherent sum of a pQCD function plus a Breit-Wigner (BW) function as

$$
\sigma^{B}(\sqrt{s})=\left|\sqrt{\frac{c_{0} \cdot \beta}{\left(\sqrt{s}-c_{1}\right)^{10}}}+e^{i \Phi} B W(\sqrt{s}) \sqrt{\frac{P(\sqrt{s})}{P(M)}}\right|^{2},
$$

is made, where $\Phi$ is a relative phase between the pQCD function and the BW function

$$
B W(\sqrt{s})=\frac{\sqrt{12 \pi \Gamma_{e e} \mathcal{B} \Gamma}}{s-M^{2}+i M \Gamma}
$$

where $M$ is mass, $\Gamma$ is width, $\Gamma_{e e}$ is the di-electron partial width, $\mathcal{B}$ is the branching fraction for the possible resonance decaying into the $\Xi^{-\bar{\Xi}^{+}}$and $\Xi^{0} \bar{\Xi}^{0}$ final states, and $P(\sqrt{s})$ is the two-body phase space factor. The significance for this possible structure decaying into the $\Xi^{-} \bar{\Xi}^{+}$final state is estimated to be $2.4 \sigma$ including the systematical uncertainties. The mass and width of the resonance around $3.0 \mathrm{GeV}$ in $e^{+} e^{-} \rightarrow \Xi^{-} \bar{\Xi}^{+}$process are fitted to be $M=(2993 \pm 28) \mathrm{MeV} / c^{2}$ and $\Gamma=(88 \pm 79) \mathrm{MeV}$, respectively, with $\Phi=2.4 \pm 0.6 \mathrm{rad}$ and $(4.3 \pm 0.3)$ rad for constructive and destructive interference conditions [37], with the statistical uncertainties only. While the mass and width of the resonance around $3.0 \mathrm{GeV}$ in the $e^{+} e^{-} \rightarrow \Xi^{0} \bar{\Xi}^{0}$ process are fixed, according to the charged mode, the significance including the systematical uncertainty is found to be $2.0 \sigma$. Thus, the upper limits on the product of the electronic partial width and the branching fractions for this possible resonance decaying to the $\Xi^{-} \bar{\Xi}^{+}$and $\Xi^{0} \bar{\Xi}^{0}$ final states are determined to be $\Gamma_{e e} \mathcal{B}<0.1(1.0)$ and $0.3 \mathrm{eV}$ at the $90 \%$ confidence level (C.L.) using a Bayesian approach [38,39], by taking into account the systematical uncertainties. In addition, the third model is also used to fit the Born cross section line shape of the $e^{+} e^{-} \rightarrow \Xi^{-\bar{G}^{+}}$process inspired by the production cross section of the nucleon pair [40] with the assumption of a near threshold plateau. It takes into account the strong interaction near the threshold instead of the Coulomb factor, and the Born cross section is expressed as:

$$
\sigma^{B}(\sqrt{s})=\frac{e^{a_{0}} \pi^{2} \alpha^{3}}{s\left[1-e^{-\frac{\pi \alpha_{s}}{\beta}}\right]\left[1+\left(\frac{\sqrt{s}-2 m_{\Xi}}{a_{1}}\right)^{a_{2}}\right]}
$$

where $a_{0}$ is the normalization constant, $a_{1}$ is the QCD parameter near threshold, $a_{2}$ is the power-law (PL) related to the number of valence quarks, and $\alpha_{s}$ is the running strongcoupling constant,

$$
\alpha_{s}=\left[\frac{1}{\alpha_{s}\left(m_{Z}^{2}\right)}+\frac{7}{4 \pi} \ln \left(\frac{s}{m_{Z}^{2}}\right)\right]^{-1} .
$$

Here $m_{Z}=91.1876 \mathrm{GeV} / \mathrm{c}^{2}$ is the mass of the $Z$ boson and $\alpha_{S}\left(m_{Z}^{2}\right)=0.11856$ is the strong coupling constant at the $Z$ pole. The inflection point of the plateau is around $3.0 \mathrm{GeV}$, which is about $350 \mathrm{MeV}$ far away from the threshold. Figure 5 shows the fitted Born cross sections for the $e^{+} e^{-} \rightarrow \Xi^{-} \bar{\Xi}^{+}$and $\Xi^{0} \bar{\Xi}^{0}$ processes. The last two assumptions are more consistent with the data than the simple perturbative QCD-driven energy power function. 
(a)

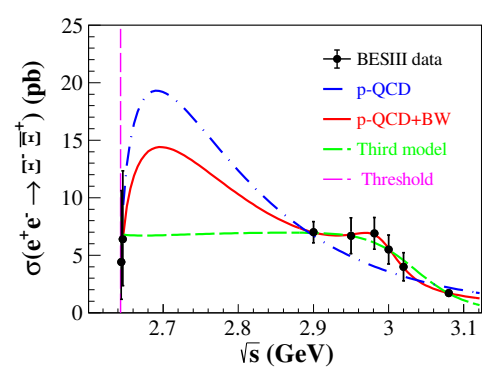

(b)

(c)
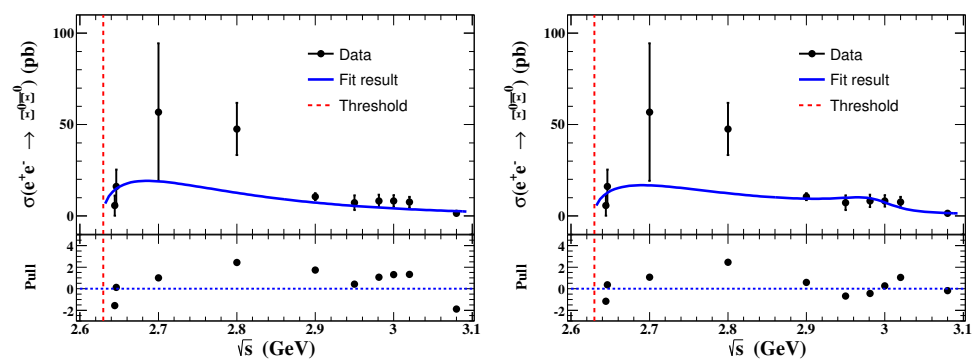

Figure 5. Fit to the Born cross sections of $e^{+} e^{-} \rightarrow \Xi^{-} \bar{\Xi}^{+}$and $\Xi^{0} \bar{\Xi}^{0}$ with different assumptions [33,34]. In (a) the dots with the error bars are the measured Born cross sections at c.m. energies between 2.644 and $3.080 \mathrm{GeV}$, the blue dash-dotted line denotes the fit results using the pQCD function, the red solid line denotes the fit results using pQCD plus the BW function and the green dashed line denotes the fit results using the third model function. In $(\mathbf{b}, \mathbf{c})$ the blue solid line is the fit result using the pQCD function (b) and pQCD plus the BW function (c), and the bottom panel of each plot gives the pull distribution of the fit. The vertical dashed line denotes the production threshold for the $\Xi$ hyperon pair.

The EFFs for $e^{+} e^{-} \rightarrow \Xi^{-} \bar{\Xi}^{+}$and $\Xi^{0} \bar{\Xi}^{0}$ are determined at each energy point, but the samples are not large enough to allow separate measurements of EMFFs, $G_{E}(s)$ and $G_{M}(s)$. The ratio of Born cross sections and EFFs for both modes is within $1 \sigma$ of the expectation of isospin symmetry as shown in Figure 6. The measured Born cross sections tend to zero around the threshold of the $\Xi$ hyperon pair production, $2.64 \mathrm{GeV}$, and show no obvious threshold enhancement. In theory, Haidenbauer et al. recently presented a phenomenological study for the EMFFs utilizing the model of the final state interaction [41]. Figure 7 shows the comparisons of Born cross section and EFF (bottom) between the experimental results of the BESIII collaboration and the theoretical calculation for the $e^{+} e^{-} \rightarrow \Xi \bar{\Xi}$ process near the threshold. It is indicated that the theoretical prediction is basically consistent with the experimental results for $e^{+} e^{-} \rightarrow \Xi^{-} \bar{\Xi}^{+}$under the current statistics [33], while it is not consistent with the experimental results between 2.64 and $2.90 \mathrm{GeV}$ for the $e^{+} e^{-} \rightarrow \Xi^{0} \bar{\Xi}^{0}$ process $[33,34]$. Figure 8 shows the theoretical predictions for the EMFFs ratio $\left|G_{E}(s) / G_{M}(s)\right|$ and the relative phase $\Phi=\arg \left(G_{E}(s) / G_{M(s)}\right)$ for the $e^{+} e^{-} \rightarrow \Xi \bar{\Xi}$ process near the threshold, which could be tested in the future when the large data sample is available. These results provide useful and important information for constraining and improving the theoretical prediction, and even offer insight into the nature of hyperon pair production in $e^{+} e^{-}$annihilation near the threshold. 


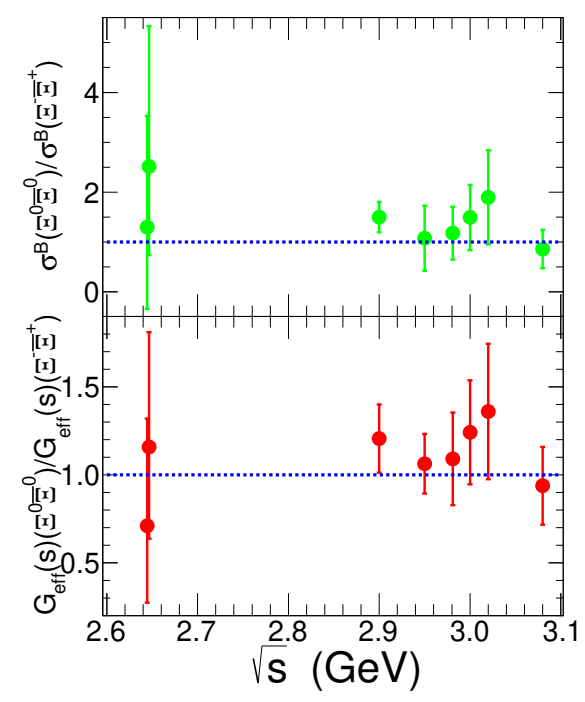

Figure 6. Ratios of Born cross sections (top) and EFFs (bottom) between the $e^{+} e^{-} \rightarrow \Xi^{-} \bar{\Xi}^{+}$mode and the $e^{+} e^{-} \rightarrow \Xi^{0} \bar{\Xi}^{0}$ mode from 2.6 to $3.1 \mathrm{GeV}[33,34]$, where the uncertainties include both statistical and systematical contributions.
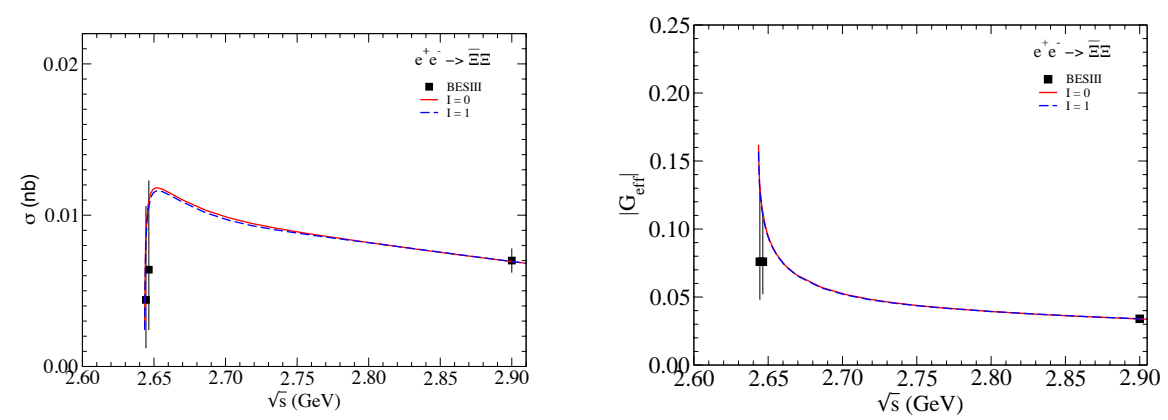

Figure 7. Comparisons of Born cross sections (left) and EFFs (right) between the experimental results of the BESIII collaboration and the theoretical study for the $e^{+} e^{-} \rightarrow \Xi \bar{\Xi}$ process near the threshold [41].
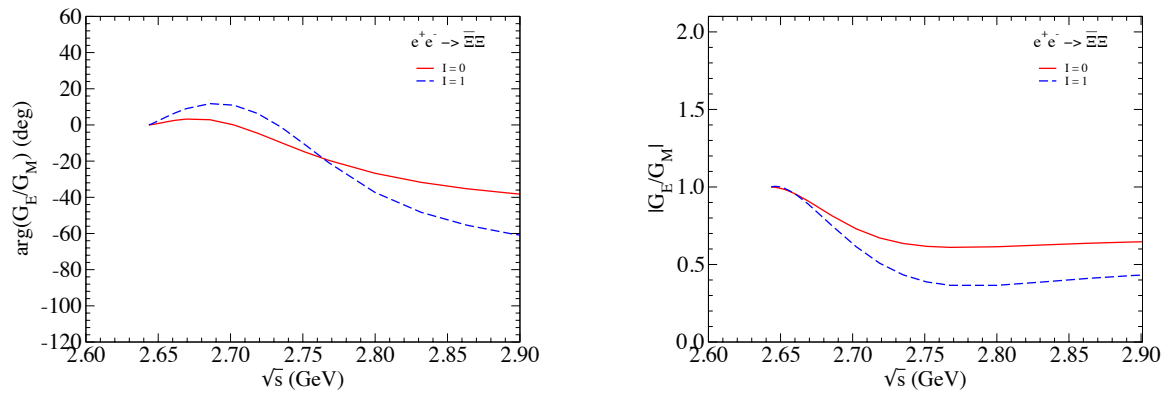

Figure 8. Theoretical predictions for the EMFFs ratio and $\left|G_{E}(s) / G_{M}(s)\right|$ and the relative phase $\Phi=\arg \left(G_{E}(s) / G_{M}(s)\right)$ for the $e^{+} e^{-} \rightarrow \Xi \bar{\Xi}$ process near the threshold [41].

\section{The $\Xi$ EMFFs above the Open Charm Threshold}

The study of EMFFs above the open charm threshold in $e^{+} e^{-}$annihilations provides important information for understanding the nature of charmonium-like states and testing the QCD calculation [42,43]. In the last decade, a series of charmonium-like states have been observed at positron-electron colliders [16]. According to potential models, as shown in Figure 9, there are more vector charmonium states between 3.77 and $4.7 \mathrm{GeV}[42,44,45]$. From experimental studies, beside the four well-established structures, i.e., $\psi(3770), \psi(4040) \psi(4160)$, and $\psi(4415)$, observed in the inclusive hadronic 
cross section [16,46], more new states, i.e., $Y(4220), Y(4360), Y(4634)$, and $Y(4660)$, have been reported in initial state radiation processes, i.e., $e^{+} e^{-} \rightarrow \gamma_{\text {ISR }} \pi^{+} \pi^{-} J / \psi$ or $e^{+} e^{-} \rightarrow$ $\gamma_{I S R} \pi^{+} \pi^{-} \psi(3686)$ in the BABAR experiment [47-50] and Belle experiment [51-55], or in direct production processes in the CLEO [56] and BESIII experiments [57-62]. However, till now, no more experimental information for hyperon production above the open charm threshold associated with these states was found, with the exception of the $\psi(4634)$ resonance observed in $\Lambda_{c}^{+} \bar{\Lambda}_{c}^{-}$by the Belle experiment [54]. The overpopulation of structures in this region and the mismatch of the properties between the experimental measurements and the potential model predictions make them good candidates for exotic states [63], such as hybrid states, tetra-quark states, or molecular states.

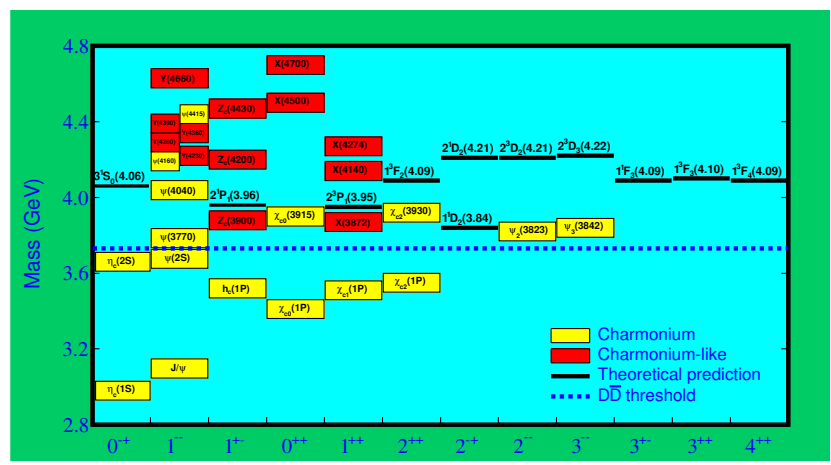

Figure 9. Charmonium(-like) family.

In 2013, the BESIII experiment performed a search for hyperonic pair production in $\psi(3770)$ and $\psi(4040)$ decays [64], including doubly-strange hyperon pair final states based on a full reconstruction method using data samples of $2.9 \mathrm{fb}^{-1}$ collected at $\sqrt{\mathrm{s}}=3.773 \mathrm{GeV}$ and $482 \mathrm{pb}^{-1}$ collected at $\sqrt{\mathrm{s}}=4.009 \mathrm{GeV}$, and no significant signals were observed. Only the upper limits for the branching fractions of $\psi(3770)$ and $\psi(4040)$ decaying into $\Xi^{-} \bar{\Xi}^{+}$ and $\Xi^{0} \bar{\Xi}^{0}$ final states at the $90 \%$ C. L. were determined to be $\mathcal{B}\left[\psi(3770) \rightarrow \Xi^{-} \bar{\Xi}^{+}\left(\Xi^{0} \bar{\Xi}^{0}\right)\right]<$ $1.5(1.4) \times 10^{-4}$ and $\mathcal{B}\left[\psi(4040) \rightarrow \Xi^{-} \bar{\Xi}^{+}\left(\Xi^{0} \bar{\Xi}^{0}\right)\right]<1.6(1.8) \times 10^{-4}$, and no EMFFs and EFF information was provided due to the limited statistics in this analysis. Subsequently, the CLEO-c experiment, by improving (by factors of 3-5) the efficiency of the hyperon identification, presented the measurements of the hyperon pair production cross sections and elastic and transition EMFFs using 805(586) $\mathrm{pb}^{-1}$ of $e^{+} e^{-}$annihilation data taken at $\sqrt{s}=3.77(4.17) \mathrm{GeV}[65,66]$. The Born cross sections and EFFs of $e^{+} e^{-} \rightarrow \Xi^{-} \bar{\Xi}^{+}$and $\Xi^{0} \bar{\Xi}^{0}$ at c.m. energy of 3.77 and $4.17 \mathrm{GeV}$ were determined as shown in Figure 10. Here, the Born cross sections for both modes are consistent with each other within $1 \sigma$ of the expectation of isospin symmetry, while the EFFs are not consistent with each other. Here the Coulomb factor $\mathcal{C}$ in Equation (9) for a neutral hyperon is different from the charged one, i.e., for a charged one, $\mathcal{C}=y /\left(1-e^{-y}\right)$ with $y=\pi \alpha \sqrt{1-\beta^{2}} / \beta$ [12-14], while for neutral one, it is unity. The CLEO-c experiment also determined the time-like form factor with the assumption $\left|G_{E} / G_{M}\right|=0$, i.e., $G_{M}(s)\left(e^{+} e^{-} \rightarrow \Xi^{-} \bar{\Xi}^{+}\right)=1.28 \pm 0.04 \pm 0.03$, $G_{M}(s)\left(e^{+} e^{-} \rightarrow \Xi^{0} \bar{\Xi}^{0}\right)=1.28 \pm 0.07 \pm 0.03$ at c.m. energy of $3.77 \mathrm{GeV}$, and $G_{M}(s)\left(e^{+} e^{-} \rightarrow\right.$ $\left.\Xi^{-} \bar{\Xi}^{+}\right)=0.44 \pm 0.06 \pm 0.01, G_{M}(s)\left(e^{+} e^{-} \rightarrow \Xi^{0} \bar{\Xi}^{0}\right)=0.40 \pm 0.08 \pm 0.04$ at c.m. energy of $4.17 \mathrm{GeV}$, as shown in Figure 11. These results achieved by the CLEO-c experiment could provide additional information to examine the features of di-quark correlations. 


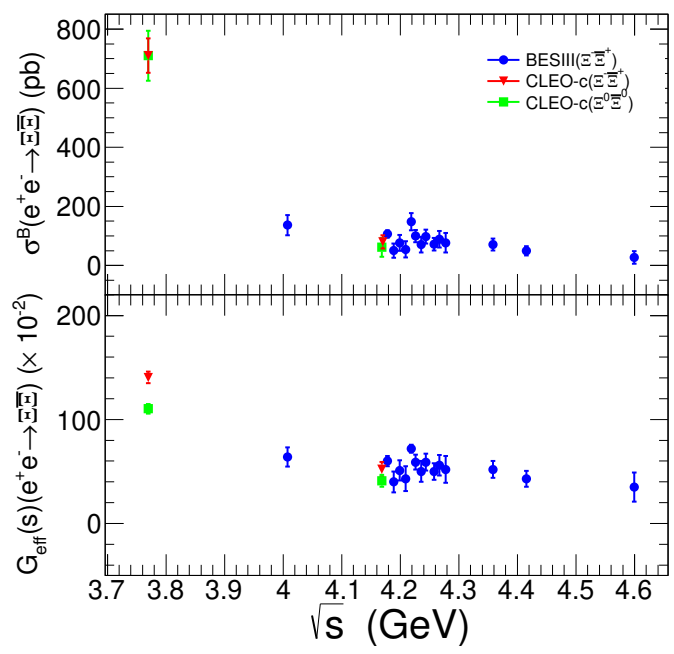

Figure 10. Comparisons of Born cross sections (top) and EFFs (bottom) between the CLEO-c experiment for the $e^{+} e^{-} \rightarrow \Xi^{-} \bar{\Xi}^{+}$and $\Xi^{0} \bar{\Xi}^{0}$ processes $[65,66]$ and the BESIII experiment for the $e^{+} e^{-} \rightarrow \Xi^{-} \bar{\Xi}^{+}$process from 3.7 to $4.6 \mathrm{GeV}$ [32], where the uncertainties include both statistical and systematical contributions.
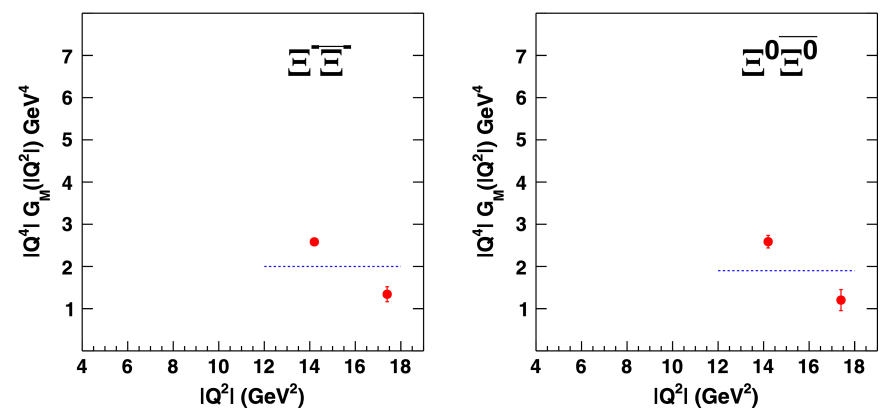

Figure 11. Illustration of $\left|Q^{2}\right|$ dependence on form factors for $e^{+} e^{-} \rightarrow \Xi^{-} \bar{\Xi}^{+}$and $\Xi^{0} \bar{\Xi}^{0}$ at c.m. energies of 3.77 and $4.17 \mathrm{GeV}[65,66]$.

Recently, BESIII collaboration also reported the measurements of Born cross sections and EFFs for the $e^{+} e^{-} \rightarrow \Xi^{-} \bar{\Xi}^{+}$process [32] with a similar method to those described for the analysis of the near threshold $[33,34]$. The dataset used in this work corresponds to a total of $11.0 \mathrm{fb}^{-1}$ of $e^{+} e^{-}$collision data [32] collected at c.m. energies from 4.009 to $4.600 \mathrm{GeV}$ with the BESIII detector at the BEPCII collider. Figure 12 shows the twodimensional distribution of $M_{\pi \Lambda}$ versus $M_{\pi \Lambda}^{\text {recoil }}$ for the sum of all energy points from 4.009 to $4.600 \mathrm{GeV}$. Clear signals for $\Xi^{-\bar{\Xi}^{+}}$production above the open charm threshold are observed for the first time.

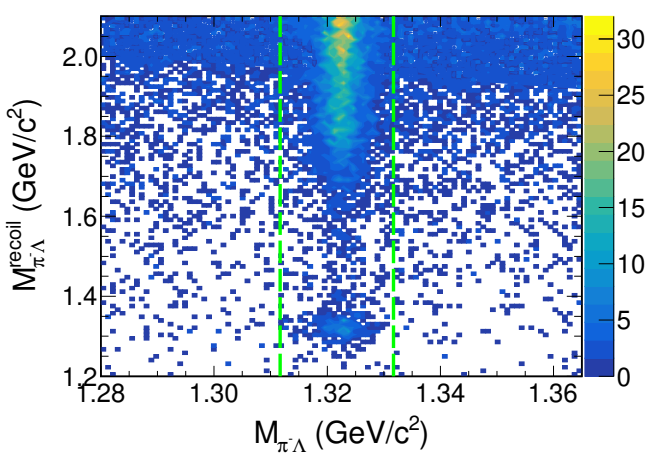

Figure 12. Distribution of $M_{\pi \Lambda}$ versus $M_{\pi \Lambda}^{\text {recoil }}$ for all energy points from data from 4.009 to $4.600 \mathrm{GeV}$ [32]. The dashed lines denote the $\Xi$ signal region. 
Figure 10 shows the comparisons of the measured Born cross sections and EFFs between the CLEO-c experiment and BESIII experiment from 4.009 to $4.600 \mathrm{GeV}$. The results for both experiments around $4.17 \mathrm{GeV}$ are consistent with each other, and are well within the uncertainty of $1 \sigma$. It should be indicated that the line shape of the cross section for the $e^{+} e^{-} \rightarrow \Xi^{-} \bar{\Xi}^{+}$process around $4.26 \mathrm{GeV}$ shows a complicated structure and could be due to the effect of some charmonium(-like) states although the uncertainties are large. In order to understand and probe the structures around $4.26 \mathrm{GeV}$ well, a maximum likelihood method is employed to fit the dressed cross section $\sigma^{\text {dressed }}=\sigma^{B} /|1-\Pi|^{2}$ for the process $e^{+} e^{-} \rightarrow \Xi^{-} \bar{\Xi}^{+}$parameterized as the coherent sum of a PL function plus a BW function for $Y(4230)$ or $Y(4260)$,

$$
\sigma^{\text {dressed }}(\sqrt{s})=\left|c_{0} \frac{\sqrt{P(\sqrt{s})}}{s^{n}}+e^{i \Phi} \mathrm{BW}(\sqrt{s}) \sqrt{\frac{P(\sqrt{s})}{P(M)}}\right|^{2},
$$

where the mass $M$, and total width $\Gamma$, are fixed to the $Y(4230) / Y(4260)$ resonance with PDG values [16], $\Phi$ is the relative phase between the BW function (see Equation (20)) and $\mathrm{PL}, n$ is a free fit parameter, and $P(\sqrt{s})$ is the two-body phase space factor. The significances of $Y(4230)$ and $Y(4260)$ resonances are found to be less than $3 \sigma$. Therefore, the upper limits on the products of the two-electron partial width and the branching fractions of $Y(4230)$ and $Y(4260) \rightarrow \Xi^{-} \bar{\Xi}^{+}$at the $90 \%$ C.L. are determined using a Bayesian approach $[38,39]$ to be $\Gamma_{e e} \mathcal{B}_{Y(4230)}<0.33 \times 10^{-3} \mathrm{eV}$ and $\Gamma_{e e} \mathcal{B}_{Y(4260)}<0.27 \times 10^{-3} \mathrm{eV}$ taking into account the systematical uncertainty, which may help understand the nature of $Y(4260)$ [67-73]. In particular, charmless decays of the $Y(4260)$ are predicted by the hybrid model [70]. Figure 13 shows the fit to the dressed cross section assuming a $Y(4230)$ or $Y(4260)$ resonance and without a resonance assumption.

(a)

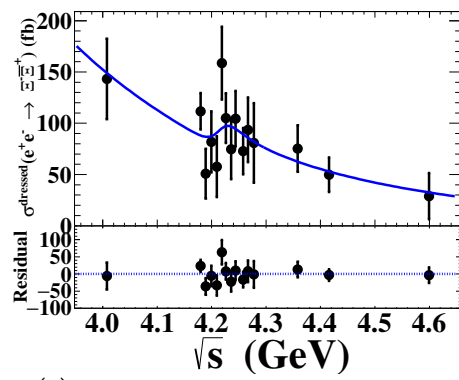

(c)

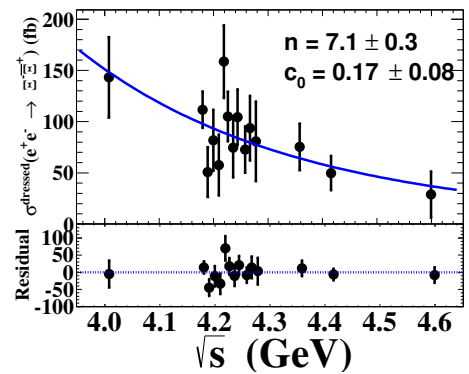

(b)

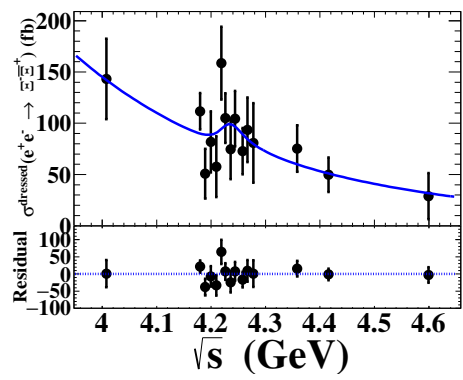

Figure 13. Fit to the dressed cross sections [32] at c.m. energies from 4.009 to $4.600 \mathrm{GeV}$ with the assumptions of a PL function plus a $Y(4230 / 4260)$ resonance function $(\mathbf{a}, \mathbf{b})$ and without a resonance assumption (c), where the dots with error bars are the dressed cross sections and the solid lines show the fit results.

Additionally, an excited $\Xi$ hyperon at $1820 \mathrm{MeV} c^{2}$ is observed by combining data of the 15 energy points in the range from 1.6 to $2.1 \mathrm{GeV} / \mathrm{c}^{2}$ [32]. The significance is estimated to be more than $6.0 \sigma$. The mass and width are measured to be $M=(1825.5 \pm 4.7 \pm 4.7) \mathrm{MeV} / c^{2}$ 
and $\Gamma=(17.0 \pm 15.0 \pm 7.9) \mathrm{MeV}$, which is consistent with the $\Xi(1820)^{-}$candidate from PDG [16] within $1 \sigma$ uncertainty, which sheds light on the structure of hyperon resonances with strangeness $S=-2$. These results also provide useful and important information for constraining and improving the theoretical prediction, and even offer insight into the nature of charmonium(-like) states and hyperon pair production in $e^{+} e^{-}$annihilation above the open charm threshold.

\section{Summary and Outlook}

In summary, we reviewed the recent studies of EMFFs on doubly-strange hyperons using the pair production near threshold and above open charm threshold reported by the BESIII experiment and CLEO-c experiment. A possible structure near $3.0 \mathrm{GeV}$ in the production cross section of $e^{+} e^{-} \rightarrow \Xi^{-} \bar{\Xi}^{+}$and $\Xi^{0} \bar{\Xi}^{0}$ is considered but the current significance is less than $3 \sigma$, which still needs be further checked when a large data sample is available. An excited $\Xi$ baryon was observed; its mass and width are consistent with the $\Xi(1820)$ baryon from PDG [16] but no quantum number was determined due to the limited statistics. These results on the $\Xi^{0}$ and $\Xi^{-}$hyperon EMFFs provide useful and important information to test the standard model, such as probing the limitation of the quark models and to spot unrevealed aspects of the QCD description of the structure of hadron resonances. It is expected that more results and surprises from the studies of the hyperon EMFFs will be made with larger data sets in $e^{+} e^{-}$annihilation in the BESIII experiment [19] and the proposed Super Tau-Charm Factory projects in China [74] and Russia [75], and the upcoming PANDA experiment in $p \bar{p}$ annihilation at FAIR [76].

Author Contributions: Conceptualization and methodology, X.W. and G.H. All authors have read and agreed to the published version of the manuscript.

Funding: This research received no external funding.

Institutional Review Board Statement: Not applicable.

Informed Consent Statement: Not applicable.

Data Availability Statement: Not applicable.

Acknowledgments: This work is supported in part by National Key Research and Development Program of China under Contract No. 2020YFA0406403; the National Natural Science Foundation of China (NSFC) under Contracts Nos. 11335008, 11905236, 12035013, 12075107, 12047501; 12061131003; the Joint Large-Scale Scientific Facility Funds of the NSFC and CAS under Contract No. U1732263; and the Fundamental Research Funds for the Central Universities under Grant No. lzujbky-2021-sp24.

Conflicts of Interest: The authors declare no conflict of interest.

\section{References}

1. Brodsky, S.J.; Farrar, G.R. Scaling laws for large-momentum-transfer processes. Phys. Rev. D 1975, 11, 1309. [CrossRef]

2. Geng, L.S.; Camalich, J.M.; Alvarez-Ruso, L.; Vicente Vacas, M.J. Leading SU(3)-breaking corrections to the baryon magnetic moments in Chiral Perturbation Theory. Phys. Rev. Lett. 2008, 101, 222002. [CrossRef]

3. Green, J.R.; Negele, J.W.; Pochinsky, A.V.; Syritsyn, S.N.; Engelhardt, M.; Krieg, S. Nucleon electromagnetic form factors from lattice QCD using a nearly physical pion mass. Phys. Rev. D 2014, 90, 074507. [CrossRef]

4. Wang, X.F. Study of baryon pair production at BESIII. PoS 2021, CHARM2020, 026.

5. Huang, G.S.; Baldini Ferroli, R. Probing the internal structure of baryons. Natl. Sci. Rev. 2021, 8, nwab187. [CrossRef] [PubMed]

6. Ablikim, M.; Achasov, M.N.; Adlarson, P.; Ahmed, S.; Albrecht, M.; Aliberti, R.; Amoroso, A.; An, M.R.; An, Q.; Bai, X.H.; et al. Measurement of $\Lambda$ baryon polarization in $e^{+} e^{-} \rightarrow \Lambda \bar{\Lambda}$ at $\sqrt{s}=3.773 \mathrm{GeV}$. arXiv 2021, arXiv:2111.11742.

7. Cabibbo, N.; Gatto, R. Electron Positron Colliding Beam Experiments. Phys. Rev. 1961, 124, 1577-1595. [CrossRef]

8. Ernst, F.J.; Sachs, R.G.; Wali, K.C. Electromagnetic form factors of the nucleon. Phys. Rev. 1960, 119, 1105-1114. [CrossRef]

9. Baldini, R.; Pacetti, S.; Zallo, A.; Zichichi, A. Unexpected featurs $E^{+} e^{-} \rightarrow P \bar{p} e^{+} e^{-} \rightarrow \Lambda \bar{\Lambda}$ cross section near threshold. Eur. Phys. J. A 2009, 39, 315-321. [CrossRef]

10. Ablikim, M.; Achasov, M.N.; Adlarson, P.; Ahmed, S.; Albrecht, M.; Aliberti, R.; Amoroso, A.; An, M.R.; An, Q.; Bai, X.H.; et al. Measurement of the Cross Section for $e^{+} e^{-} \rightarrow \Lambda \bar{\Lambda}$ and Observation of the Decay $\psi(3770) \rightarrow \Lambda \bar{\Lambda}$. Phys. Rev. D 2021, 104, L091104. [CrossRef]

11. Arbuzov, A.B.; Kopylova, T.V. On relativization of the Sommerfeld-Gamow-Sakharov factor. JHEP 2012, 4, 009. [CrossRef] 
12. Sommerfeld, A. Über die Beugung und Bremsung der Elektronen. Ann. Phys. 1931, 403, 257. [CrossRef]

13. Sakharov, A.D. Interaction of an Electron and Positron in Pair Production. Phys. Usp. 1991, 34, 375. [CrossRef]

14. Tzara, C. Effect of the coulomb potential on the $\pi^{-}$photoproduction. Nucl. Phys. B 1970, 18, 246-252. [CrossRef]

15. Baldini Ferroli, R.; Pacetti, S.; Zallo, A. No Sommerfeld resummation factor in $e^{+} e^{-} \rightarrow p \bar{p}$. Eur. Phys. J. A 2012, 48, 33. [CrossRef]

16. Zyla, P.A.; Barnett, R.M.; Beringer, J.; Bonventre, R.; Dahl, O.; Dwyer, D.A.; Groom, D.E.; Lin, C.J.; Lugovsky, K.S.; Pianori, E.; et al. Review of Particle Physics. Prog. Theor. Exp. Phys. 2020, 2020, 083 C01.

17. Available online: https://commons.m.wikimedia.org/wiki/Category:Baryon_multiplets (accessed on 1 October 2021).

18. Yu, C.; Duan, Z.; Gu, S.; Guo, Y.; Huang, X.; Ji, D.; Ji, H.; Jiao, Y.; Liu, Z.; Peng, Y. BEPCII Performance and Beam Dynamics Studies on Luminosity. In Proceedings of the IPAC2016, Busan, Korea, 8-13 May 2016. [CrossRef]

19. Ablikim, M.; Achasov, M.N.; Adlarson, P.; Ahmed, S.; Albrecht, M.; Alekseev, M.; Amoroso, A.; An, F.F.; An, Q.; Bai, Y.; et al. Future Physics Programme of BESIII. Chin. Phys. C 2020, 44, 040001. [CrossRef]

20. Naik, P.; CLEO Collaboration. Advances in Open Charm Physics at CLEO-c. Nucl. Phys. B Proc. Suppl. 2011, 210-211, 137-140. [CrossRef]

21. Pedlar, T.K. The CLEOIII detector. Int. J. Mod. Phys. A 2001, 16S1C, 1059-1061.

22. Aubert, B.; Bona, M.; Boutigny, D.; Karyotakis, Y.; Lees, J. P.; Poireau, V.; Prudent, X.; Tisserand, V.; Zghiche, A.; Garra Tico, J.; et al. Study of $e^{+} e^{-} \rightarrow \Lambda \bar{\Lambda}, \Lambda \bar{\Sigma}^{0}, \Sigma^{0} \bar{\Sigma}^{0}$ using initial state radiation with BABAR. Phys. Rev. D 2007, 76, 092006. [CrossRef]

23. Bisello, D.; Busetto, G.; Castro, A.; Nigro, M.; Pescara, L.; Posocco, M.; Sartori, P.; Stanco, L.; Antonelli, A.; Biagini, M.E.; et al. Baryon pair production in $e^{+} e^{-}$annihilation at $\sqrt{s}=2.4 \mathrm{GeV}$. Z. Phys. C 1990, 48, 23-28.

24. Ablikim, M.; Achasov, M.N.; Ahmed, S.; Ai, X.C.; Albayrak, O.; Albrecht, M.; Ambrose, D.J.; Amoroso, A.; An, F.F.; An, Q.; et al Observation of a cross-section enhancement near mass threshold in $e^{+} e^{-} \rightarrow \Lambda \bar{\Lambda}$. Phys. Rev. D 2018, 97, 032013. [CrossRef]

25. Ablikim, M.; Achasov, M.N.; Ahmed, S.; Albrecht, M.; Alekseev, M.; Amoroso, A.; An, F.F.; An, Q.; Bai, J.Z.; Bai, Y.; et al. Precision measurement of the $e^{+} e^{-} \rightarrow \Lambda_{c}^{+} \bar{\Lambda}_{c}^{-}$cross section near threshold. Phys. Rev. Lett. 2018, 120, 132001. [CrossRef]

26. Ablikim, M.; Achasov, M.N.; Albayrak, O.; Ambrose, D.J.; An, F.F.; An, Q.; Bai, J.Z.; Bai, Y.; Ban, Y.; Becker, J.; et al. Measurements of baryon pair decays of $\chi_{c J}$ mesons. Phys. Rev. D 2013, 87, 032007. [CrossRef]

27. Ablikim, M.; Achasov, M.N.; Ahmed, S.; Ai, X.C.; Albayrak, O.; Albrecht, M.; Ambrose, D.J.; Amoroso, A.; An, F.F.; An, Q.; et al. Study of $\psi$ decays to the $\Xi^{-} \bar{\Xi}^{+}$and $\Sigma(1385)^{\mp} \bar{\Sigma}(1385)^{ \pm}$final states. Phys. Rev. D 2016, 93, 072003. [CrossRef]

28. Ablikim, M.; Achasov, M.N.; Ahmed, S.; Ai, X.C.; Albayrak, O.; Albrecht, M.; Ambrose, D.J.; Amoroso, A.; An, F.F.; An, Q.; et al. Study of $J / \psi$ and $\psi(3686) \rightarrow \Sigma(1385)^{0} \bar{\Sigma}(1385)^{0}$ and $\Xi^{0} \bar{\Xi}^{0}$. Phys. Lett. B 2017, 770, 217-225. [CrossRef]

29. Ablikim, M.; Achasov, M.N.; Adlarson, P.; Ahmed, S.; Albrecht, M.; Alekseev, M.; Amoroso, A.; An, F.F.; An, Q.; Bai, Y; et al. Observation of $\psi(3686) \rightarrow \Xi(1530)^{-} \bar{\Xi}(1530)^{+}$and $\Xi(1530)^{-} \bar{\Xi}^{+}$. Phys. Rev. D 2019, 100, 051101. [CrossRef]

30. Wang, X.F.; Li, B.; Gao, Y.N.; Lou, X.C. Helicity amplitude analysis of $J / \psi$ and $\psi(3686) \rightarrow \Xi(1530) \bar{\Xi}(1530)$. Nucl. Phys. B 2019, 941, 861-867. [CrossRef]

31. Ablikim, M.; Achasov, M.N.; Adlarson, P.; Ahmed, S.; Albrecht, M.; Alekseev, M.; Amoroso, A.; An, F.F.; An, Q.; Bai, Y.; et al. Measurement of $J / \psi \rightarrow \Xi(1530)^{-} \bar{\Xi}^{+}$and evidence for the radiative decay $\Xi(1530)^{-} \rightarrow \gamma \Xi^{-}$. Phys. Rev. D 2020, 101, 012004. [CrossRef]

32. Ablikim, M.; Achasov, M.N.; Adlarson, P.; Ahmed, S.; Albrecht, M.; Alekseev, M.; Amoroso, A.; An, F.F.; An, Q.; Bai, Y.; et al. Measurement of the cross section for $e^{+} e^{-} \rightarrow \Xi^{-} \bar{\Xi}^{+}$and observation of an excited $\Xi$ baryon. Phys. Rev. Lett. 2020, 124, 032002 . [CrossRef]

33. Ablikim, M.; Achasov, M.N.; Adlarson, P.; Ahmed, S.; Albrecht, M.; Aliberti, R.; Amoroso, A.; An, Q.; Bai, X.H.; Bai, Y; et al. Measurement of cross section for $e^{+} e^{-} \rightarrow \Xi^{-} \bar{\Xi}^{+}$near threshold at BESIII. Phys. Rev. Lett. 2021, 103, 012005. [CrossRef]

34. Ablikim, M.; Achasov, M.N.; Adlarson, P.; Ahmed, S.; Albrecht, M.; Aliberti, R.; Amoroso, A.; An, Q.; Bai, X.H.; Bai, Y; et al. Measurement of cross section for $e^{+} e^{-} \rightarrow \Xi^{0} \bar{\Xi}^{0}$ near threshold. Phys. Lett. B 2021, 820, 136557. [CrossRef]

35. Pacetti, S.; Baldini Ferroli, R.; Tomasi Gustafsson, E. Proton electromagnetic form factors: Basic notions, present achievements and future perspectives. Phys. Rep. 2015, 550, 1. [CrossRef]

36. Ablikim, M.; Achasov, M.N.; Adlarson, P.; Ahmed, S.; Albrecht, M.; Amoroso, A.; An, Q.; Anita,; Bai, Y; Bakina, O.; et al. Measurements of $\Sigma^{+}$and $\Sigma^{-}$time-like electromagnetic form factors for center-of-mass energies from 2.3864 to $3.0200 \mathrm{GeV}$. Phys. Lett. B 2021, 814, 136110. [CrossRef]

37. Zhu, K.; Mo, X.H.; Yuan, C.Z.; Wang, P. A mathematical review on the multiple-solution problem. Int. J. Mod. Phys. A 2011, 26, 4511-4520. [CrossRef]

38. Zhu, Y.S. Bayesian credible interval construction for Poisson statistics. Chin. Phys. C 2008, 32, 363.

39. D'Agostini, G. Bayesian Reasoning in Data Analysis: A Critical Introduction; World Scientific: Hackensack, NJ, USA, 2003 ; p. 329.

40. Ablikim, M.; Achasov, M.N.; Adlarson, P.; Ahmed, S.; Albrecht, M.; Alekseev, M.; Amoroso, A.; An, F.F.; An, Q.; Anita,; et al. Measurement of proton electromagnetic form factors in $e^{+} e^{-} \rightarrow p \bar{p}$ in the energy region 2.00-3.08 GeV. Phys. Rev. Lett. 2020, 124, 042001. [CrossRef]

41. Haidenbauer, J.; Meißner, U.G.; Dai, L.Y. Hyperon electromagnetic form factors in the timelike region. Phys. Rev. D 2021, 103, 014028. [CrossRef]

42. Brambilla, N.; Eidelman, S.; Heltsley, B.K.; Vogt, R.; Bodwin, G.T.; Eichten, E.; Frawley, A.D.; Meyer, A.B.; Mitchell, R.E. Papadimitriou, V.; et al. Heavy Quarkonium: Progress, Puzzles, and Opportunities. Eur. Phys. J. C 2011, 71, 1534. [CrossRef] 
43. Briceno, R.A.; Cohen, T.D.; Coito, S.; Dudek, J.J.; Eichten, E.; Fischer, C.S.; Fritsch, M.; Gradl, W.; Jackura, A.; Kornicer, M.; et al. Issues and Opportunities in Exotic Hadrons. Chin. Phys. C 2016, 40, 042001. [CrossRef]

44. Godfrey, S.; Isgur, N. Mesons in a Relativized Quark Model with Chromodynamics. Phys. Rev. D 1985, 32, 189-231. [CrossRef]

45. Yuan, C.Z. Charmonium and Charmoniumlike States at the BESIII Experiment. arXiv 2021, arXiv:2102.12044.

46. Wang, X.F. Experimental Status of Conventional Charmonium Spectroscopy. EPJ Web Conf. 2019, 202, 02002. [CrossRef]

47. Aubert, B.; Barate, R.; Boutigny, D.; Couderc, F.; Karyotakis, Y.; Lees, J.P.; Poireau, V.; Tisserand, V.; Zghiche, A.; Grauges, E.; et al. Observation of a broad structure in the $\pi^{+} \pi^{-} J / \psi$ mass spectrum around $4.26 \mathrm{GeV} / \mathrm{c}^{2}$. Phys. Rev. Lett. 2005, 95, 142001. [CrossRef]

48. Aubert, B.; Barate, R.; Bona, M.; Boutigny, D.; Couderc, F.; Karyotakis, Y.; Lees, J.P.; Poireau, V.; Tisserand, V.; Zghiche, A.; et al. Evidence of a broad structure at an invariant mass of $4.32 \mathrm{GeV} / \mathrm{c}^{2}$ in the reaction $e^{+} e^{-} \rightarrow \pi^{+} \pi^{-} \psi_{2 S}$ measured at BaBar. Phys. Rev. Lett. 2007, 98, 212001. [CrossRef]

49. Lees, J.P.; Poireau, V.; Tisserand, V.; Garra Tico, J.; Grauges, E.; Palano, A.; Eigen, G.; Stugu, B.; Brown, D.N.; Kerth, L.T.; et al. Study of the reaction $e^{+} e^{-} \rightarrow J / \psi \pi^{+} \pi^{-}$via initial-state radiation at BaBar. Phys. Rev. D 2012, 86, 051102. [CrossRef]

50. Lees, J.P.; Poireau, V.; Tisserand, V.; Garra Tico, J.; Grauges, E.; Palano, A.; Eigen, G.; Stugu, B.; Brown, D.N.; Kerth, L.T.; et al. Study of the reaction $e^{+} e^{-} \rightarrow \psi(2 S) \pi^{-} \pi^{-}$via initial-state radiation at BaBar. Phys. Rev. D 2014, 89, 111103. [CrossRef]

51. Yuan, C.Z.; Shen, C.P.; Wang, P.; McOnie, S.; Adachi, I.; Aihara, H.; Aulchenko, V; Aushev, T.; Bahinipati, S.; Barberio, E.; et al Measurement of $e^{+} e^{-} \rightarrow \pi^{+} \pi^{-} J / \psi$ cross-section via initial state radiation at Belle. Phys. Rev. Lett. 2007, 99, 182004. [CrossRef]

52. Wang, X.L.; Yuan, C.Z.; Shen, C.P.; Wang, P.; Adachi, I.; Aihara, H.; Arinstein, K; Aushev, T.; Bakich, A.M.; Barberio, E.; et al. Observation of Two Resonant Structures in $e^{+} e^{-} \rightarrow \pi^{+} \pi^{-} \psi(2 S)$ via Initial State Radiation at Belle. Phys. Rev. Lett. 2007, 99, 142002. [CrossRef] [PubMed]

53. Wang, X.L.; Yuan, C.Z.; Shen, C.P.; Wang, P.; Abdesselam,A.; Adachi, I.; Aihara, H.; Al Said, H.; Arinstein, K; Asner, D.M.; et al. Measurement of $e^{+} e^{-} \rightarrow \pi^{+} \pi^{-} \psi(2 S)$ via Initial State Radiation at Belle. Phys. Rev. D 2015, 91, 112007. [CrossRef]

54. Pakhlova, G.; Adachi, I.; Aihara, H.; Al Said, H.; Arinstein, K.; Aulchenko, V.; Bakich, A.M.; Barberio, E.; Bedny, I.; Bhardwaj, V.; et al. Observation of a near-threshold enhancement in the $e^{+} e^{-} \rightarrow \Lambda_{c}^{+} \Lambda_{c}^{-}$cross section using initial-state radiation. Phys. Rev. Lett. 2008, 101, 172001. [CrossRef]

55. Liu, Z.Q.; Yuan, C.Z.; Shen, C.P.; Adachi, I.; Aihara, H.; Asner, D.M.; Aulchenko, V.; Aushev, T.; T. Aziz, T.; Bakich, A.M.; et al. Study of $e^{+} e^{-} \rightarrow \pi^{+} \pi^{-} J / \psi$ and Observation of a Charged Charmoniumlike State at Belle. Phys. Rev. Lett. 2013, 110, 252002. [CrossRef]

56. Coan, T.E.; Gao, Y.S., Liu, F.; Artuso, M.; Blusk, S.; Butt, J.; Li, J.; Menaa, N.; Mountain, R.; Nisar, S.; et al. Charmonium decays of $Y(4260), \psi(4160)$ and $\psi(4040)$. Phys. Rev. Lett. 2006, 96, 162003. [CrossRef]

57. Ablikim, M.; Achasov, M.N.; Ai, X.C.; Albayrak, O.; Albrecht, M.; Ambrose, D.J.; Amoroso, A.; An, F.F.; An, Q.; Bai, J.Z.; et al Study of $e^{+} e^{-} \rightarrow \omega \chi_{c J}$ at center-of-mass energies from 4.21 to $4.42 \mathrm{GeV}$. Phys. Rev. Lett. 2015, 114, 092003. [CrossRef] [PubMed]

58. Ablikim, M.; Achasov, M.N.; Ahmed, S.; Ai, X.C.; Albayrak, O.; Albrecht, M.; Ambrose, D.J.; Amoroso, A.; An, F.F.; An, Q.; et al. Precise measurement of the $e^{+} e^{-} \rightarrow \pi^{+} \pi^{-} J / \psi$ cross section at center-of-mass energies from 3.77 to $4.60 \mathrm{GeV}$. Phys. Rev. Lett. 2017, 118, 092001. [CrossRef] [PubMed]

59. Ablikim, M.; Achasov, M.N.; Ahmed, S.; Albrecht, M.; Alekseev, M.; Amoroso, A.; An, F.F.; An, Q.; Bai, J.Z.; Bakina, O.; et al Measurement of $e^{+} e^{-} \rightarrow \pi^{0} \pi^{0} \psi(3686)$ at $\sqrt{s}$ from 4.009 to $4.600 \mathrm{GeV}$ and observation of a neutral charmoniumlike structure. Phys. Rev. D 2018, 97, 052001. [CrossRef]

60. Ablikim, M.; Achasov, M.N.; Adlarson, P.; Ahmed, S.; Albrecht, M.; Alekseev, M.; Amoroso, A.; An, F.F.; An, Q.; Bai, Y.; et al. Cross section measurement of $e^{+} e^{-} \rightarrow \eta^{\prime} J / \psi$ from $\sqrt{s}=4.178$ to $4.600 \mathrm{GeV}$. Phys. Rev. D 2020, 101, 012008. [CrossRef]

61. Ablikim, M.; Achasov, M.N.; Adlarson, P.; Ahmed, S.; Albrecht, M.; Alekseev, M.; Amoroso, A.; An, F.F.; An, Q.; Bai, Y.; et al. Study of the process $e^{+} e^{-} \rightarrow \pi^{0} \pi^{0} J / \psi$ and neutral charmonium-like state $Z_{c}(3900)^{0}$. Phys. Rev. D 2020, 102, 012009. [CrossRef]

62. Ablikim, M.; Achasov, M.N.; Adlarson, P.; Ahmed, S.; Albrecht, M.; Aliberti, R.; Amoroso, A.; An, M.R.; An, Q.; Bai, X.H.; et al. Cross Section Measurement of $e^{+} e^{-} \rightarrow \pi^{+} \pi^{-} \psi(3686)$ from $\sqrt{s}=4.0076 \mathrm{GeV}$ to $4.6984 \mathrm{GeV}$. Phys. Rev. D 2021, $104,052012$. [CrossRef]

63. Chen, H.X.; Chen, W.; Liu, X.; Zhu, S.L. The hidden-charm pentaquark and tetraquark states. Phys. Rept. 2016, 639, 1-121. [CrossRef]

64. Ablikim, M.; Achasov, M.N.; Albayrak, O.; Ambrose, D.J.; An, F.F.; An, Q.; Bai, J.Z.; Bai, Y.; Baldini Ferroli, R.; Ban, Y.; et al. Search for baryonic decays of $\psi(3770)$ and $\psi(4040)$. Phys. Rev. D 2013, 87, 112011. [CrossRef]

65. Dobbs, S.; Tomaradze, A.; Xiao, T.; Seth, K.K.; Bonvicini, G. First measurements of timelike form factors of the hyperons, $\Lambda^{0}$, $\Sigma^{0}$, $\Sigma^{+}, \Xi^{0}, \Xi^{-}$, and $\Omega^{-}$, and evidence of diquark correlations. Phys. Lett. B 2014, 739, 90-94. [CrossRef]

66. Dobbs, S.; Seth, K.K.; Tomaradze, A.; Xiao, T.; Bonvicini, G. Hyperon Form Factors and Diquark Correlations. Phys. Rev. D 2013, 96, 092004. [CrossRef]

67. Maiani, L.; Riquer, V.; Piccinini, F.; Polosa, A.D. Four quark interpretation of Y(4260). Phys. Rev. D 2005, 72, 031502. [CrossRef]

68. Liu, X.; Zeng, X.Q.; Li, X.Q. Possible molecular structure of the newly observed Y(4260). Phys. Rev. D 2005, 72, 054023. [CrossRef]

69. Zhu, S.L. The possible interpretations of $Y(4260)$. Phys. Lett. B 2005, 625, 212. [CrossRef]

70. Close, F.E.; Page, P.R. Gluonic charmonium resonances at BaBar and BELLE. Phys. Lett. B 2005, 628, 215-222. [CrossRef]

71. Wang, Z.G. Analysis of the vector tetraquark states with P-waves between the diquarks and antidiquarks via the QCD sum rules. Eur. Phys. J. C 2019, 79, 29. [CrossRef] 
72. Dubnička, S.; Dubničková, A.Z.; Issadykov, A.; Ivanov, M.A.; Liptaj, A. Y(4260) as a four-quark state. Phys. Rev. D 2020, 101, 094030. [CrossRef]

73. Wang, J.Z.; Qian, R.Q.; Liu, X.; Matsuki, T. Are the $Y$ states around $4.6 \mathrm{GeV}$ from $e^{+} e^{-}$annihilation higher charmonia? Phys. Rev. $D$ 2020, 101, 034001. [CrossRef]

74. Zhou, X.R.; STCF Working Group. Experimental Program at Super Tau-Charm Facility. PoS 2021, CHARM2020, 007.

75. Bondar, A.E.; Project of a Super Charm-Tau factory at the Budker Institute of Nuclear Physics in Novosibirsk. Phys. Atom. Nucl. 2013, 76, 1072-1085. [CrossRef]

76. Belias, A. FAIR status and the PANDA experiment. JINST 2020, 15, C10001. [CrossRef] 